\title{
Transforming Transmission Electron Microscopy with MerlinEM Electron Counting Detector
}

Matus Krajnak ${ }^{1}$, Jean-Denis Blazit ${ }^{2}$, Alexander Zintler ${ }^{3}$, Robert Eilhardt ${ }^{3}$, Jon Weiss ${ }^{4}$, Doug Cosart ${ }^{4}$, Leopoldo Molina-Luna ${ }^{3}$, Marcel Tencé ${ }^{2}$, Alexandre Gloter ${ }^{2}$, Christopher Allen $^{5}$ and Damien McGrouther ${ }^{6}$

${ }^{1}$ Quanum Detectors Ltd., Harwell Oxford, England, United Kingdom, ${ }^{2}$ Université Paris-Saclay, CNRS, Orsay, Ile-de-France, France, ${ }^{3}$ Technical University of Darmstadt, Darmstadt, Hessen, Germany, ${ }^{4}$ NanoMEGAS USA, Tempe, Arizona, United States, ${ }^{5}$ Electron Physical Science Imaging Centre, Diamond Light Source, Didcot, England, United Kingdom, ${ }^{6}$ University of Glasgow, UK, Glasgow, Scotland, United Kingdom

At the University of Glasgow, experiments with hybrid pixelated counting detectors started with a Medipix2 detector [1]. It was clear that direct electron detection and hardware-based electron counting can offer advantageous imaging capabilities. Subsequently, a Medipix3 detector with Merlin readout system was commercialised as a MerlinEM detector by a collaboration between the University of Glasgow and Quantum Detectors Ltd. The detector has now been applied in multiple experimental configurations. In this paper capabilities in 4D-STEM, electromagnetic field imaging, ptychography, precession electron diffraction and electron energy loss spectroscopy will be demonstrated.

The MerlinEM detector has been applied to 4D-STEM at the prototype stage to improve magnetic imaging in Lorentz microscopy [2]. It was shown that acquiring a full diffraction pattern for each point in a scan with subsequent gradient correlation processing vastly improves signal to noise. The sensitivity of this new method allowed imaging of magnetic fields with the highest spatial resolution available on the microscope $(<1 \mathrm{~nm}$ field free imaging on probe corrected Jeol ARM200 cFEG at University of Glasgow) which was not previously accessible using the standard (annular) quadrant detection. The Fig. 1(a) shows a colour image showing a quantitative integrated magnetic induction map from $10 \mathrm{~nm}$ thick permalloy sample imaged with a $200 \mathrm{kV}$ probe with convergence semi-angle of $2.15 \mathrm{mrad}$. The deflection of the probe is in the range of $<0.3$ pixels across the different magnetic domains.

The benefits of electron counting, and dynamic range binning, can be exploited for electron ptychography. The MerlinEM detector can be used with up to $18800 \mathrm{fps}$ in 1-bit (binary) mode, to achieve atomic resolution in very low dose regime [3] and can offer significant advantages for sample stability and charging issues. Ptychography is ideally suited for imaging of 2D materials; a reconstruction of a phase of twisted bilayer graphene sample is shown in Fig 1(b). The data has been taken and processed at ePSIC with a Jeol ARM300CF operated at $60 \mathrm{kV}$ and MerlinEM detector. It is apparent that ptychography offers higher signal to noise if compared with a simultaneously collected ADF image in Fig 1(c).

Direct electron detection together with the higher number of pixels (256 x 256 or $512 \times 512)$ and the high dynamic range ( $\max 24$-bit counting) can be used in conjunction for improved results with precession electron diffraction. NanoMEGAS SPRL has integrated the MerlinEM camera into their imaging framework. Direct detection enhances the speed, stability and reliability of the precession diffraction toolkit. We will present recent new results on this work.

The EELS version purposes the MerlinEM in a 4x1 configuration (1024 x 256). It has been installed at STEM LPS Orsay lab on a Cs corrected Nion USTEM200 microscope using a Gatan ENFINA spectrometer. Apart from the high DQE and MTF, there are two main advantages of the detector for EELS: high dynamic range (24-bit) for collection of the zero loss peak area; and zero read-out noise for collecting the high loss edges with high signal to noise ratio. An example is given in Fig. 2 where spectrum imaging was performed over an STO/LMO interface [4] with $100 \mathrm{kV}$ illumination, 250pA beam current and $10 \mathrm{~ms}$ dwell time. The spectrometer was set to capture core loss Ti-K and La-L edges between $4.5-5.7 \mathrm{kV}$. 
(a)

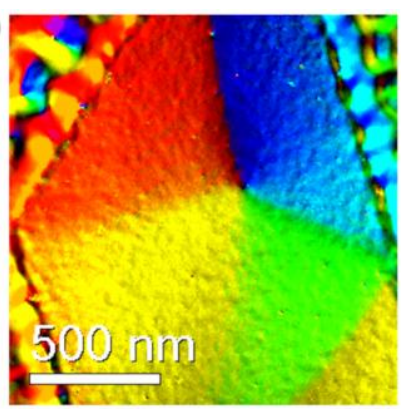

(b)

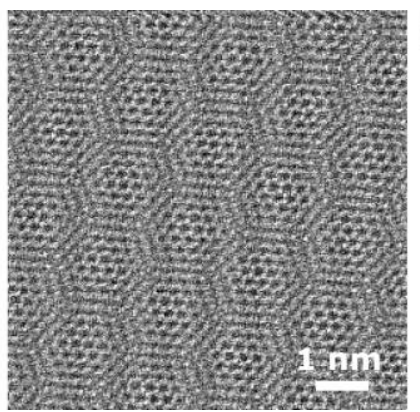

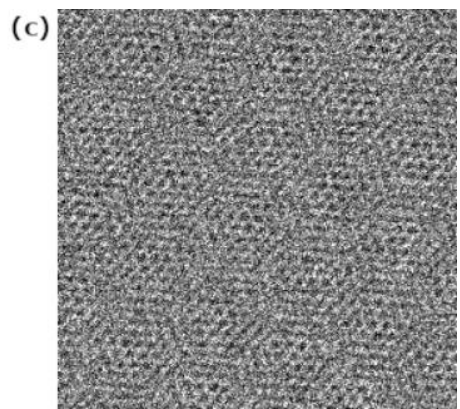

Figure 1. (a) Quantitative DPC imaging of integrated magnetic induction in $10 \mathrm{~nm}$ thick permalloy sample, probe convergence semi-angle was $2.15 \mathrm{mrad}$. The orientation and magnitude of the induction is shown in the colour wheel. (b) and (c) reconstructed phase and simultaneously collected ADF of a twisted graphene bilayer sample. Imaging was performed at ePSIC with Jeol ARM300CF operated at $60 \mathrm{kV}$.

(a)

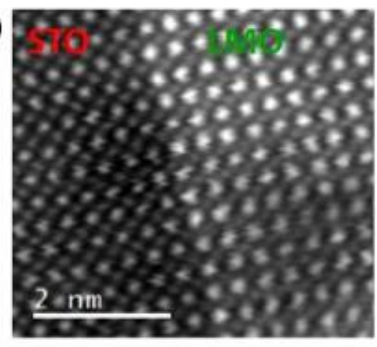

(b)

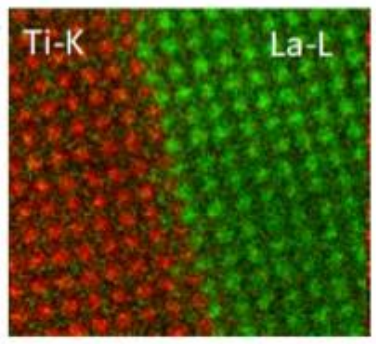

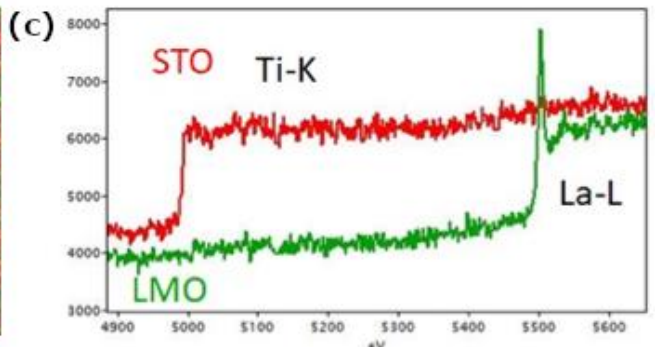

Figure 2. Figure 2: (a) HAADF collected along EELS acquisition, (b) high core loss Ti-K and La-L EELS atomically resolved map collected by MerlinEM 4x1 detector, (c) comparison of the spectra from STO and LMO showing distinct separation of the two edges. Imaging was done at $100 \mathrm{kV}$ with $250 \mathrm{pA}$ beam current with Nion USTEM200 and Gatan ENFINA spectrometer.

\section{References}

[1] Mac Raighne, A., et al. "Medipix2 as a highly flexible scanning/imaging detector for transmission electron microscopy." Journal of Instrumentation 6.01 (2011): C01047.

[2] Krajnak, Matus, et al. "Pixelated detectors and improved efficiency for magnetic imaging in STEM differential phase contrast." Ultramicroscopy 165 (2016): 42-50.

[3] O'Leary, Colum M., et al. "Electron Ptychography Using Fast Binary 4D STEM Data." Microscopy and Microanalysis 25.S2 (2019): 1662-1663.

[4] Gibert, Marta, et al. "Interfacial control of magnetic properties at LaMnO3/LaNiO3 interfaces." Nano letters 15.11 (2015): 7355-7361. 